\title{
CLINICAL IMPORTANCE AND REPRESENTATION OF TOXIGENIC AND NON-TOXIGENIC CLOSTRIDIUM DIFFICILE CULTIVATED FROM STOOL SAMPLES OF HOSPITALIZED PATIENTS
}

\author{
Stojanovic Predrag ${ }^{1,2 *}$, Kocic Branislava ${ }^{1,2}$, Stojanovic Miodrag ${ }^{1,3}$, Miljkovic - Selimovic Biljana ${ }^{1,2}$, Tasic Suzana ${ }^{1,2}$, \\ Miladinovic - Tasic Natasa ${ }^{1,2}$, Babic Tatjana ${ }^{2}$
}

${ }^{1}$ Faculty of Medicine, University of Nis, Serbia; ${ }^{2}$ Institute for Public Health Nis, Center of Microbiology, Serbia; ${ }^{3}$ Institute for Public Health Nis, Center of Biostatistics and medical informatics, Serbia.

Submitted: May 11, 2010; Returned to authors for corrections: March 18, 2011; Approved: August 30, 2011.

\begin{abstract}
The aim of this study was to fortify the clinical importance and representation of toxigenic and non-toxigenic Clostridium difficile isolated from stool samples of hospitalized patients. This survey included 80 hospitalized patients with diarrhea and positive findings of Clostridium difficile in stool samples, and 100 hospitalized patients with formed stool as a control group.

Bacteriological examination of a stool samples was conducted using standard microbiological methods. Stool sample were inoculated directly on nutrient media for bacterial cultivation (blood agar using 5\% sheep blood, Endo agar, selective Salmonella Shigella agar, Selenite-F broth, CIN agar and Skirrow's medium), and to selective cycloserine-cefoxitin-fructose agar (CCFA) (Biomedics, Parg qe tehnicologico, Madrid, Spain) for isolation of Clostridium difficile. Clostridium difficile toxin was detected by ELISA-ridascreen Clostridium difficile Toxin A/B (R-Biopharm AG, Germany) and ColorPAC ToxinA test (Becton Dickinson, USA). Examination of stool specimens for the presence of parasites (causing diarrhea) was done using standard methods (conventional microscopy), commercial concentration test Paraprep S Gold kit (Dia Mondial, France) and RIDA ${ }^{\circledR}$ QUICK Cryptosporidium/Giardia Combi test (R-Biopharm AG, Germany). Examination of stool specimens for the presence of fungi (causing diarrhea) was performed by standard methods. All stool samples positive for Clostridium difficile were tested for Rota, Noro, Astro and Adeno viruses by ELISA - ridascreen (R-Biopharm AG, Germany).

In this research we isolated 99 Clostridium difficile strains from 116 stool samples of 80 hospitalized patients with diarrhea. The $53(66.25 \%)$ of patients with diarrhea were positive for toxins A and B, one (1.25\%) were positive for only toxin B. Non-toxigenic Clostridium difficile isolated from samples of 26 (32.5\%) patients. However, other pathogenic microorganisms of intestinal tract cultivated from samples of 16 patients. Examination of cultivated colonies revealed that most of cultivated species belonged to genera of Campylobacter spp., Salmonella spp., and Candida spp.. In control group, toxigenic Clostridium difficile cultivated from stool samples of two patients (2\%) and non-toxigenic Clostridium difficile from samples of five patients (5\%).

This research confirmed clinical importance of toxigenic Clostridium difficile found in liquid stool samples of hospitalized patient, and the possibility of asymptomatic carriage in $2 \%$ of patients with formed stool.
\end{abstract}

Key words: Clostridium difficile, diarrhea, toxins

*Corresponding Author. Mailing address: The Faculty of Medicine in Nis, Serbia.; E-mail: pedjamicro@bankerinter.net 


\section{INTRODUCTION}

Clostridium (C.) difficile is an anaerobic, gram - positive, spore forming bacteria, is commonly present in soil and add intestinal tract of animals, healthy children and adults. Intrahospital infections caused by $C$. difficile are commonly related to contaminated surfaces of facilities or by the unclean hands of medical staff (17).

By the opinion of numerous medical researchers $(3,4,7)$ there are toxigenic and non-toxigenic $C$. difficile strains. Toxigenic $C$. difficile, found by in intestine of healthy persons as a component of normal microbiota, is not a health risk (asymptomatic carriage) (12), but in persons with risk factors (antibiotics usage, elderly patients, long-term hospitalization, multiple comorbid conditions, cytostatic, x-ray, immunosuppressive and corticosteroid therapy etc.) (21) it could cause diarrhea of different severities, up to lifethreatening pseudomembranous colitis (PMC). Disease caused by $C$. difficile itself is known as Clostridium difficile associated disease - CDAD and could be randomly found in hospitalized patients, though it may outbreaks in patients admitted to intensive care units (21). Typical clinical manifestations of CDAD include abdominal pain and cramps, profuse diarrhea (slimy, dirty greenish, watery stool with highly unpleasant odor), fever, and leukocytosis (4).

Antibiotics therapy causes disbalance of intestinal tract bacterial flora, which predisposes $C$. difficile proliferation and colonization of intestinal tract mucosa (7). Afterwards, toxigenic $C$. difficile may excrete exotoxins, enterotoxin (toxin A) and citotoxin (toxin B), and both can cause diarrhea and colitis. Toxigenic $C$. difficile can produce simultaneously both toxins $(\mathrm{A}+\mathrm{B}+)$. The results obtained during the last fifteen years suggest that strains of $C$. difficile that excrete only toxin $\mathrm{B}(\mathrm{A}-\mathrm{B}+)$ have clinical significance $(1,3,6)$.

Results of many studies, conducted over the past twenty years, have shown that toxigenic strains were present in about 60 to $90 \%$ of patients with diarrhea and C. difficile $(4,17,24)$. In microbiological laboratories across Europe, among toxigenic strains, the most frequently cultivated $\mathrm{A}+\mathrm{B}+$ strain (up to $97 \%$ )
(21). The prevalence of $C$. difficile $\mathrm{A}-/ \mathrm{B}+$ strain is higher in other parts of the World, such as Japan (39\%), Korea (43\%), and Israel (56\%). These strains are most dominant in Argentina $(97.9 \%)$, regarding to total number of toxigenic isolates $(6,11$, 14, 22, 23). All these surveys confirmed that toxigenic strains of $C$. difficile, cultivated from stool samples of hospitalized patient with diarrhea, have clinical importance and require appropriate therapy, that very often includes metronidazole and/or vancomycin. There are different opinions about nontoxigenic (A-/B-) C. difficile clinical importance (12), but prevailing view is that it mostly belong to normal microbiota of intestinal tract. However, results of several studies indicate that non-toxigenic $C$. difficile are present in stool of hospitalized patients with persistent diarrhea, whose stool samples did not present other pathogenic microorganisms (bacteria, viruses, parasites, and fungi) of intestinal tract $(9,18)$.

According to available data from referent literature, until the present day, the investigation of CDAD has not been conducted in Serbia, and this is the first research considering of this health problem.

The aim of the study is determination of clinical importance and representation of toxigenic and non-toxigenic Clostridium difficile presence in stool samples of hospitalized patients.

\section{MATERIALS AND METHODS}

Research was performed at the Institute of Public Health Nis - Serbia, Center for Microbiology, during the period from December 2006 - August 2009. Clinical trial group included 80 hospitalized patients with diarrhea. The selection of patients was based on following criteria:

- $\quad$ Presence of at least three unformed or liquid stools during $24 \mathrm{~h}$ for at least 2 days.

- Hospitalization, at least $48 \mathrm{~h}$ before the appearance of unformed or liquid stool.

- Cultivation of C. difficile (toxigenic and non-toxigenic strains) from examined stool samples.

The control group consisted of 100 hospitalized patients with formed stool, without signs and symptoms of 
intestinal tract infections. Patients of control group were hospitalized, for at least $48 \mathrm{~h}$, in different clinics of Clinical center in Nis.

In this prospective study we processed data of clinical trial and control group patients. Data were collected from the microbiological laboratories (type of isolated microorganism, production of toxin) and from clinics of Clinical Centers from three cities in Serbia (sex, age, therapy applied before diarrhea: antibiotics, cytostatics, corticosteroids, and x-ray therapy; number of stools within the last $24 \mathrm{~h}$, duration of diarrhea, high temperature peak values and their duration, number of leukocytes, blood electrolyte concentration (potassium and sodium), administration of therapy against CDAD). Daily lab reports (labor list, Lab protocols) about isolation of pathogens in microbiological laboratories and medical documentation (histories, temperature lists) were used as a source data.

Standard microbiological procedure was applied during bacteriological examination of a stool samples. Stool sample were directly inoculated on nutrient media for bacterial cultivation (blood agar using 5\% sheep blood, Endo agar, selective Salmonella Shigella agar, Selenite-F broth, CIN agar and Skirrow's medium), as well as, selective cycloserinecefoxitin-fructose agar (CCFA) (Biomedics, Parg qe tehnicologico, Madrid, Spain) for $C$. difficile cultivation after alcohol-shock procedure application. Adequate incubation period preceded the identification of colonies, which was performed by standard microbiological methods.

$\mathrm{CCF}$ agar was incubated at $37^{\circ} \mathrm{C}$ under anaerobic conditions for $48 \mathrm{~h}$. AnaeroGen sachets (OXOID, England) were used to anaerobic condition in jars. Anaerobic strips (Anaerobic indicator, OXOID, England) were used to verify anaerobic conditions. Commercial API system for anaerobic bacteria (API 20A BioMerieux, France) was applied for biochemical identification of $C$. difficile isolates (typical colonies were $4 \mathrm{~mm}$ or larger in diameter, elevated, convex, with a discrete margin, an irregular surface and strong horse manure-like odor).

C. difficile toxins A and B was detected in stool specimens by ELISA-ridascreen Clostridium difficile Toxin A/B ( $\mathrm{R}$ Biopharm AG, Germany). C. difficile toxine A was detected in stool specimens by ColorPAC Toxin A test (Becton Dickinson, USA). Colonies of $C$. difficile were subcultivated in $5 \mathrm{ml}$ brainheart infusion broth under anaerobic conditions during four days. After incubation, liquid cultures of $C$. difficile were centrifugated at $3000 \mathrm{~g}$ for $15 \mathrm{~min}$. and filtred trought $0.45 \mu \mathrm{m}$ membranes. Determination of toxins was performed using previosly cited tests according to manufacturer's instruction. Same procedure was applied on liquid cultures of reference strains $C$. difficile ATCC $43598(\mathrm{~A}-/ \mathrm{B}+)$ and $C$. difficile ATCC $43255(\mathrm{~A}+/ \mathrm{B}+)$ cultivated in brain-heart infusion broth under anaerobic conditions within four days.

Examination of stool specimens for a detection of protozoa and helminthes was performed by conventional microscopy and commercial concentration test Paraprep S Gold kit (Dia Mondial, France). Presence of antigens of Cryptosporidium parvum and Giardia lamblia in stool samples from patients was determined by RIDA $^{\circledR}$ QUICK Cryptosporidium/Giardia Combi test (R-Biopharm AG, Germany). Examination of stool specimens for detection of fungi was performed by standard methods (fungi cultivation on Sabouraud agar plates at $37^{\circ} \mathrm{C}$, within 5 - 7 days under aerobic atmosphere). Candida albicans was differentiated from non-albicans species by germinative tube production test and based on growth of colored colonies on chromatogenic medium (Chromogen albicans - Biomedics, Parg qe tehnicologico, Madrid, Spain).

All stool samples positive for $C$. difficile were tested for Rota, Noro, Astro and Adeno viruses by ELISA - ridascreen (R-Biopharm AG, Germany).

Diagnosis of CDAD was based on a presence of the following criteria (12)

- Diarrheal stools or toxic megacolon, and a positive laboratory assay for $C$. difficile toxin A and/or toxin B in stools or a toxin-producing $C$. difficile detected in stool via culture or other means.

SPSS (version 15) statistical package was used for 
statistical analysis. Data were presented as mean \pm standard deviation. Parametric Student t-test for independent samples and nonparametric Mann Whitney $U$ test were applied for the comparison of two group values, while ANOVA test was performed for 3 subgroups data comparison. P-value which was less than 0.05 was considered as significant.

\section{RESULTS}

We isolated 99 C. difficile strains from 116 stool samples of 80 hospitalized patients with diarrhea during this research. Patients' age ranged from 4 - 81 years (52.50\% male, and $47.50 \%$ female). ELISA-ridascreen Clostridium difficile Toxin $\mathrm{A} / \mathrm{B}$ test was positive for toxin presence in stool samples of 54 patients $(67.50 \%)$ with diarrhea.

The $66.25 \%$ of patients with diarrhea were positive for toxins $\mathrm{A}$ and $\mathrm{B}(\mathrm{A}+\mathrm{B}+), 1.25 \%$ were positive for only toxin $\mathrm{B}$ $(\mathrm{A}-/ \mathrm{B}+)$, while $32.5 \%$ were negative for both toxins $(\mathrm{A}-/ \mathrm{B}-)$ (Table 1).

Table 1. Production of toxin A and B of Clostridium difficile isolated from stools samples of hospitalized patients with diarrhea.

\begin{tabular}{|c|c|c|c|c|c|c|}
\hline \multirow{2}{*}{$\begin{array}{l}\text { Production the certain toxin of } \\
\text { Clostridium difficile }\end{array}$} & \multirow{2}{*}{$\begin{array}{c}\text { Find of Elisa- } \\
\text { ridascreen Toxin } \\
\text { A/B test } \\
\end{array}$} & \multirow{2}{*}{$\begin{array}{c}\text { Find of } \\
\text { ColorPAC } \\
\text { Toxin A test } \\
\end{array}$} & \multirow{2}{*}{$\begin{array}{c}\text { Number of stool } \\
\text { samples }\end{array}$} & \multirow[t]{2}{*}{ Number of isolates } & \multicolumn{2}{|c|}{ Number of patients } \\
\hline & & & & & $\mathbf{n}$ & $\%$ \\
\hline excrete toxin $\mathrm{A}$ and $\mathrm{B}(\mathrm{A}+\mathrm{B}+)$ & + & + & 77 & 69 & 53 & 66.25 \\
\hline excrete only toxin $\mathrm{B}(\mathrm{A}-/ \mathrm{B}+)$ & + & - & 2 & 2 & 1 & 1.25 \\
\hline Non-toxigenic (A-/B-) & - & - & 37 & 28 & 26 & 32.50 \\
\hline TOTAL & & & 116 & 99 & 80 & 100 \\
\hline CONTROL & & & & & & \\
\hline ATCC43598 (A-/B+) & + & - & & & & \\
\hline ATCC43255 (A+/B+) & + & + & & & & \\
\hline
\end{tabular}

Presence of toxigenic $C$. difficile was determined in stool samples of $90.74 \%$ patients with diarrhea, who were previously treated with antibiotics (from 7 to 35 days). Cytostatic therapy, was administered to four patients with diarrhea and toxigenic C. difficile. However, beside 30 days long cytostatic therapy, two patients were treated with antibiotics due to urinary tract infection (7 and 8 days), too. Pronison therapy (15 days) preceded diarrhea in one patient.

Non-toxigenic $C$. difficile (A-/B-) was cultivated from the samples obtained from $26(32.5 \%)$ patients with diarrhea. Evaluation of results in this group of patients showed: a) in stool samples of 16 patients, other pathogenic microorganisms of intestinal tract were detected (the most frequently cultivated species are Campylobacter jejuni, Salmonella enterica serovar Typhimurium and Candida albicans); b) from stool samples of 5 patients few colonies $(2-4)$ of normal intestinal microbiota were cultivated; c) in last five patients, from stool samples non- toxigenic $C$. difficile was cultivated, too, but the all other cultivation media were sterile (Table 2).

Table 2. Pathogenic microorganisms associated with Clostridium difficile non-toxigenic strains (A-/B-) isolated from stool samples of hospitalized patients with diarrhea.

\begin{tabular}{lcr}
\hline \multirow{2}{*}{ Species of microorganisms } & \multicolumn{2}{c}{$\begin{array}{c}\text { Number of } \\
\text { patients }\end{array}$} \\
\hline all other cultivation media were sterile (subgroup 3) & 5 & 19.23 \\
Campilobacter jejuni & 4 & 15.38 \\
Candida albicans & 4 & 15.38 \\
Salmonella enterica serovar Typhimurium & 3 & 11.53 \\
normal intestinal microbiota (subgroup 1) & 3 & 11.53 \\
normal intestinal microbiota (subgroup 2) & 2 & 7.70 \\
Rota virus & 2 & 7.70 \\
Salmonella enterica serovar Enteritidis & 1 & 3.85 \\
Geotrichum candidum & 1 & 3.85 \\
Cryptosporidium parvum & 1 & 3.85 \\
\hline TOTAL & 26 & 100 \\
\hline
\end{tabular}


Analysis of collected data from medical history, confirmed that the following groups of patients received antibiotic therapy: a) patients with non-toxigenic $C$. difficile and normal intestinal microbiota (from 7 to 10 days; one or two antibiotics); b) patients with Candida albicans isolates (from 14 to 19 days; two or more different antibiotics) c) patients with non-toxigenic $C$. difficile and all other cultivation media sterile (from 17 to 21 days; two or more different antibiotics).

Metronidazole treatment was conducted (within 4 to 11 days, 8 days in average) to the 54 patients with toxigenic $C$. difficile as diarrhea treatment. The eight of 54 patients, after metronidazole treatment, continued to take vancomycin (from 4 to 6 days).

From the two patients with non-toxigenic $C$. difficile and normal intestinal microbiota, diarrhea was not cured until metronidasole was administrated (6 - 8 days). In the rest of three patients with non-toxigenic $C$. difficile and normal intestinal microbiota, the only way of treating was to stop antibiotic therapy previously prescribed. After quitting antibiotic therapy, diarrhea ended spontaneously. That was also the case of 5 patients with non-toxigenic $C$. difficile and the all other cultivation media sterile.

Appropriate etiologic therapy was applied in patients with confirmed presence of intestinal pathogenic species (Campylobacter jejuni., Salmonella enterica serovar Typhimurium and serovar Enteritidis, Candida albicans etc.),

One hundred stool samples of hospitalized patients from control group (57\% male, and 43\% female; age ranged from 14 to 84 years), were also examined. Toxigenic $C$. difficile was cultivated from the stool samples of two patients $(2 \%)$. Presence of non-toxigenic $C$. difficile was confirmed in samples of five patients (5\%). Toxigenic isolates of $C$. difficile were positive for toxins $\mathrm{A}$ and $\mathrm{B}(\mathrm{A}+\mathrm{B}+)$. In patients from control group, applied methodology did not reveal presence of any other intestinal tract pathogenic microorganisms in examined stool samples.

Statistical analysis showed that in patients with diarrhea and toxigenic $C$. difficile strains isolates, number of leukocytes, number of stools within 24 hours, body temperature, blood sodium concentration was significantly different $(p<0.05)$ in relation to the control group (patients without $C$. difficile and without diarrhea).

During this research, we were specifically focused on clinical parameters for the following subgroup of patients:

- Three patients with normal intestinal microbiota isolates and non-toxigenic $C$. difficile, where diarrhea spontaneously stopped after they finished antibiotic therapy previously prescribed (subgroup 1).

- Two patients with normal intestinal microbiota isolates and non-toxigenic $C$. difficile, with metronidazole therapy for diarrhea (subgroup 2).

- Five patients with diarrhea and non-toxigenic $C$. difficile, with all other cultivated media sterile (subgroup 3).

Statistical analysis of evaluated parameters from these subgroups patients $(1,2$, and 3$)$, compared of the control group (patients without $C$. difficile and without diarrhea) showed that there is statistically significant difference in the number of stools within 24 hours and sodium concentration. The most interesting is the subgroups 2 , which number of stools, number of leukocytes and body temperature were significantly different compared the same parameters of the control group (patients without $C$. difficile and without diarrhea). Values of some parameters (duration of diarrhea, number of leukocytes and body temperature) were significantly higher in subgroup 2 compared the same parameters of subgroups 1 and 3. Also, duration of the high temperature peak in subgroup 2 were significantly higher in relation to the subgroup 1. Statistical difference was not found in observed parameters between subgroups 1 and 3 (Table 3 ). 
Table 3. The relation between the clinical parameters of the control group pacients and the pacients with diarrhea and nontoxigenic $C$. difficile (subgroup 1, 2 and 3)

\begin{tabular}{|c|c|c|c|c|c|c|c|c|c|}
\hline \multirow{2}{*}{$\begin{array}{l}\text { Group and subgroup } \\
\text { Parameters }\end{array}$} & \multicolumn{2}{|c|}{$\begin{array}{c}\text { Pacients of control } \\
\text { group (CG) - without } \\
\begin{array}{c}\text { C. difficile and without } \\
\text { diarrhea }\end{array} \\
n=93\end{array}$} & \multicolumn{2}{|c|}{$\begin{array}{c}\text { Patients with } \\
\text { diarrhea and non- } \\
\text { toxigenic } C . \\
\text { difficile } \\
\text { (subgroup 1) } \\
\text { n = 3 } \\
\end{array}$} & \multicolumn{2}{|c|}{$\begin{array}{c}\text { Patients with } \\
\text { diarrhea and non- } \\
\text { toxigenic } C \text {. } \\
\text { difficile } \\
\text { (subgroup 2) } \\
\text { n=2 } \\
\end{array}$} & \multicolumn{2}{|c|}{$\begin{array}{c}\text { Patients with } \\
\text { diarrhea and } \\
\text { non-toxigenic } C \text {. } \\
\text { difficile } \\
\text { (subgroup 3) } \\
\text { n = 5 } \\
\end{array}$} & \multirow[b]{2}{*}{$\operatorname{Sig}(\mathrm{p}<0,05)$} \\
\hline & $\bar{X}$ & SD & $\bar{X}$ & $\mathrm{SD}$ & $\bar{X}$ & SD & $\bar{X}$ & SD & \\
\hline Number of stools $/ 24^{\mathrm{h}}$ & 1.43 & 0.53 & 3.33 & 0.57 & 7.50 & 0.70 & 4.20 & 0.83 & A,B,C,D \\
\hline $\begin{array}{l}\text { Duration of diarrhea } \\
\text { (in days; } 1 \text { day }=24^{\mathrm{h}} \text { ) }\end{array}$ & - & - & 4.00 & 1.00 & 7.50 & 0.70 & 3.20 & 0.83 & $\mathrm{D}, \mathrm{F}$ \\
\hline Number of leukocytes/ $\mu \mathrm{L}$ & 7.11 & 2.46 & 6.93 & 0.25 & 12.15 & 0.77 & 7.06 & 0.47 & $\mathrm{~B}, \mathrm{D}, \mathrm{F}$ \\
\hline $\begin{array}{l}\text { Concentracion of } \\
\text { potassium } \mathrm{mmol} / \mathrm{L}\end{array}$ & 4.10 & 0.12 & 4.06 & 0.05 & 3.95 & 0.07 & 4.04 & 0.13 & NS \\
\hline $\begin{array}{l}\text { Concentracion of sodium } \\
\mathrm{mmol} / \mathrm{L}\end{array}$ & 139.71 & 3.81 & 131.67 & 2.51 & 126.0 & 2.82 & 128.60 & 2.19 & $\mathrm{~A}, \mathrm{~B}, \mathrm{C}$ \\
\hline Body temperature $\left({ }^{\circ} \mathrm{C}\right)$ & 37.00 & 0.16 & 37.20 & 0.10 & 38.20 & 0.42 & 37.42 & 0.38 & $\mathrm{~B}, \mathrm{D}, \mathrm{F}$ \\
\hline $\begin{array}{l}\text { Duration of the high body } \\
\text { temperature peak } \\
\text { (in days; } 1 \text { day }=24^{\mathrm{h}} \text { ) }\end{array}$ & - & - & 1.00 & 0.00 & 2.50 & 0.70 & 1.40 & 0.54 & $\mathrm{D}$ \\
\hline
\end{tabular}

\section{DISCUSSION}

Though there are documents about PMC since 1893, $C$. difficile was described for the first time in 1935 by Hall and O'Toole (10). It was considered as a non-pathogenic, due to the fact that it was commonly cultivated from stool samples of healthy children. In the late 1970 s, it was confirmed that $C$. difficile is toxigenic bacteria that can cause intestinal tract diseases (13).

Evaluation of numerous surveys indicated the presence of toxigenic and non-toxigenic $C$. difficile in different geographic areas. In European hospitals toxigenic strains were dominant among $C$. difficile strains isolated from stool samples of patients with diarrhea (12). Research of Malamou-Lada $\mathrm{H}$. showed that isolates of $C$. difficile were non-toxigenic (A-/B-) in $7 \%$, toxigenic $\mathrm{A}-\mathrm{B}+$ in $3 \%$, and $\mathrm{A}+\mathrm{B}+$ in $90 \%$ patients with diarrhea (16). Paltansing S. et al. (18) reported that $C$. difficile was cultivated from stool samples of 81 hospitalized patients with diarrhea (87\% A+/B+, 10\% A-/B- and 3\% A-/B+). Higher percentage $(25.77 \%)$ of non-toxigenic $C$. difficile strains was proved in research of Gayane M. et al. (9). However, in this survey, toxigenic $C$. difficile $(\mathrm{A}+\mathrm{B}+)$ isolates were more frequent $(69.07 \%)$ than those that produce only toxin B (A$/ \mathrm{B}+)(5.16 \%)$. The results of this study conducted in Serbia showed that isolates of $C$. difficile found in 80 patients with diarrhea (66.25\% $\mathrm{A}+/ \mathrm{B}+, 1.25 \% \mathrm{~A}-/ \mathrm{B}+$ and $32.50 \% \mathrm{~A}-/ \mathrm{B}-)$. The presented data show the dominance of toxigenic isolates, especially $\mathrm{A}+/ \mathrm{B}+$ strains. During the last ten years, $\mathrm{A}-/ \mathrm{B}+C$. difficile strains were cultivated in several countries. Prevalence of $0.2 \%$ was recorded in USA (15), in the United Kingdom is $3 \%$ (5) and France is 3\% (2). Discovery of A-/B+C. difficile strains had great importance for the diagnosis of CDAD. Most microbiology laboratories, in routine work, using reagents that are determining the presence of toxin A only, and their use results a non - detect $\mathrm{A}-\mathrm{B}+$ strains (5). For this reason have been developed reagents, which simultaneously determines the presence of toxins A and B (15).

The causes of differences in the prevalence of toxigenic 
and non-toxigenic $C$. difficile in patients with diarrhea are not fully understood. According to previously cited research papers $(5,9,15,16,18)$, the prevalence of toxigenic and nontoxigenic strains is different, because studies were conducted in different time periods (one or more years), in different geographical areas and included different population groups (children and / or adults and / or persons over 60 years). The results of research done in Serbia can be compared to the study of Guyana M. et al. (9) (the similar investigated clinical groups). However, it must be emphasized that $C$. difficile strains could be present in intestine of hospitalized patient with formed stool. The results of prospective studies show that among hospitalized patients of Western Europe, the carriage rate of $C$. difficile (toxigenic and non-toxigenic) varied from 5.9 to $11 \%(4,21)$. The rate of carriers was even higher in elderly patients hospitalized in intensive care wards (14\%) and in elderly patients $(20 \%)$ who were hospitalized for a long time because of chronic illness (19). It is higher rate of carriers than in healthy adults (3\%) (21), and can be caused by the use of antibiotics and colonization of intestinal tract during previous hospitalizations. In our study prevalence of asymptomatic carriage of $C$. difficile (toxigenic and non-toxigenic) strains was $7 \%$ in hospitalized patients with formed stool. The prevalence of toxigenic strains only is $2 \%$.

All researchers $(4,7,12,18)$ agree that cultivation of toxigenic $C$. difficile strains without presence of other intestinal pathogenic microorganisms, in liquid stool samples, is clinically important finding. There are many studies performed during 1970s, where the significance of $\mathrm{A}+\mathrm{B}+$ strain of $C$. difficile as causative agent of diarrhea and colitis was confirmed $(4,7,17)$. Likewise, the published results proved that $\mathrm{A}-\mathrm{B}+$ strains can cause $\mathrm{CDAD}$ in sporadic and epidemic form $(1,5,23)$. Survey conducted in Serbia confirms that A$/ \mathrm{B}+C$. difficile has the ability to cause disease, because this strain was found in patient with diarrhea.

There are different opinions about clinical importance of non-toxigenic (A-/B-) C. difficile (12). Prevailing view is that it mostly belong to normal microbiota of intestinal tract and that it is not a pathogen microorganism $(3,4,7)$. Findings of other enteropathogens (bacteria, viruses, parasites, and fungi) in liquid stool samples along with non-toxigenic strains of $C$. difficile support this theory.

Interpretation of positive non-toxigenic $C$. difficile strains findings in stool samples of patients with diarrhea and understanding of its role in this condition is very difficult, because many of patients during the investigation were on antibiotic treatment. Diarrhea can be an outcome of antibiotic intake (antibiotic-associated diarrhea - AAD). Most of the researches (25) agree upon the fact that antibiotic-associated diarrhea occurs as a result of imbalance of normal bacterial microbiota in intestine and due to osmotic effect of nonabsorbed carbohydrate. During an antibiotic therapy these normal metabolic processes are disturbed which leads to the retention of carbohydrate and results in an osmotic diarrhea. There is a lack of systemic symptoms, and in most patients diarrhea stops after the ceasing of antibiotic use. Results of our research correlate with this theory. Patients with diarrhea and non-toxigenic $C$. difficile (subgroup 1 and 3), had diarrhea after antibiotic therapy. The therapy included to two antibiotics in a shorter period of time (patients of subgroup 1) or broadspectrum antibiotics for a longer period (patients of subgroup 3 ). The diarrhea spontaneously stooped in patients from these two subgroups after the ceasing of antibiotics intake, which indicates the presence of AAD.

However, there are several studies that indicate clinical importance of non-toxigenic $C$. difficile. Hence, Paltansing S. et al. (18), described non-toxigenic isolate obtained from hospitalized patient with diarrhea. Gayane M. et al. $(8,9)$ described 4 patients with non-toxigenic $C$. difficile isolate accompanied with leukocytosis, increased body temperature and diarrhea that lasted 6 to 10 days. Researchers $(8,9,18)$ indicated that non-toxigenic strains may contain some other virulence factors such as adhesins, enzymes, capsules, flagella, antibiotic resistance etc, that can play a role in colonization and 
disease development. Numerous researchers $(12,20)$ indicated that some toxigenic strains of $C$. difficile $(1-8 \%)$ produce a binary toxin (ADP- Ribosyltransferase), beside toxins A and B. The exact role of this toxin remains unclear, but there is evidence that binary toxin, similarly to toxins A and B, affect actin and that leads to damage of cytoskeleton. Clinical relevance of binary toxin was additionally supported by the discovery of non-toxigenic $C$. difficile (A-/B-) which produce binary toxin (20). The results obtained by our study in Serbia show that non-toxigenic isolates (obtained from two patients from subgroup 2) maybe the cause of diarrhea. Further investigation with a larger patient population and more modern microbiological methodology is necessary to better understand the role that non-toxigenic $C$. difficile strains play in disease development.

The study conducted in Serbia also confirms clinical importance of toxigenic $C$. difficile found in liquid stool samples of hospitalized patients, and the possibility of asymptomatic carriage in $2 \%$ of patients with formed stool.

\section{REFERENCES}

1. Alfa, M.; al-Barrak, A.; Embil, J.; Dyck, B.; Olekson, K.; Nicoll, D. (1999). An outbreak of toxin A negative, toxin B positive Clostridium difficile-associated diarrhea in a Canadian tertiary-care hospital. Can. Commun. Dis. Rep. 25 (7): 65-69.

2. Barbut, F. ; Lalande, V. ; Burghoffer, B. ; Thien, H.V. ; Grimprel, E. ; Petit, J.C. (2002). Prevalence and genetic characterization of toxin A variant strains of Clostridium difficile among adults and children with diarrhea in France. J. Clin. Microbiol. 40(6): 2079-2083.

3. Borriello, S.P. (1998). Pathogenesis of Clostridium difficile infection. J. Antimicrob. Chemother. 41: 13 - 19

4. Bouza, E.; Munoz, P.; Alonso, R. (2005). Clinical manifestations, tretment and control of infections caused by Clostridium difficile. Clin. Mic. Infec. Dis. 11: 57 - 64.

5. Brazier, J.; Stubbs, S.; Duerden. B.I. (1999). Prevalence of toxin A negative/B positive Clostridium difficile strains. J. Hosp. Infect. 42(3): 248-249.

6. Drudy, D.; Fanning, S.; Kyne, L. (2007). Toxin A-negative, toxin Bpositive Clostridium difficile. Int. J. Infect. Dis. 11(1): 5-10.

7. Farrell, R.J.; LaMont, J.T. (2000) Pathogenesis and clinical manifestations of Clostridium difficile diarrhea and colitis. Curr. Topics. Microbiol. Immunol. 250: 109 - 125.

8. Gayane, M.; Adam, S.; Joseph, S. (2005). Clostridium difficile in emergency room. Anaerobe. 11: 258-261.

9. Gayane, M.; Stuart, C.; Szczesny, A.; Silva, J. (2005). Analysis of Clostridium difficile-associated diarrhea among patients hospitalized in tertiary academic hospital. Diag. Microb. Infec. Dis. 52: 153 - 155.

10. Hall, I.C.; Tool, E. (1935). Intestinal flora in newborn infants with a description of a new pathogenic anaerobe, Bacillus difficilis. Am. J. Dis. Child. 49: 390-402.

11. Komatsu, M.; Kato, H.; Aihara, M.; Shimakawa, K.; Iwasaki, M.; Nagasaka, Y. (2003). High frequency of antibiotic-associated diarrhea due to toxin A-negative, toxin B-positive Clostridium difficile in a hospital in Japan and risk factors for infection. Eur. J. Clin. Microbiol. Infect. Dis. 22(9): 525-529.

12. Kuijper, E.J.; Coignard, B.; Tull, P. (2006). Emergence of Clostridium difficile-associated disease in North America and Europe. Clin. Microbiol. Infect. 12: 2-18.

13. Larson, H.E.; Paryy, J.V.; Price, A.B.; Davies, D.R.; Tyrell, D.A.; Dolby, J. (1977). Undescribed toxin in pseudomembraneus colitis. Br. Med. J. 1: 1246 - 1248 .

14. Legaria, M.C.; Rossetii, S.; Lumelsky, G. (2003). Clostridium difficileassociated diarrhea from a general hospital in Argentina. Anaerobe. 9: $113-116$.

15. Lyerly, D.; Neville, L.; Evans, D.; Fill, J.; Allen, S.; Greene, W. (1998). Multicenter evaluation of the Clostridium difficile TOX A/B TEST. $J$. Clin. Microbiol. 36(1): 184-190.

16. Malamou-Lada, H.; Kafkoula-Karafilidis, P.; Strouza, A. (2005). Toksin $\mathrm{A}(-) /$ toksin $\mathrm{B}(+)$ Clostridium difficile strains isolated from patients with diarrhoea in a tertiary hospitall in Athens. $15^{\text {th }}$ European congres of Clinical microbiology and infectionus diseases; Copenhagen/Denmark, $\mathrm{p}$. 1135 .

17. McFarland, L.V.; Stamm, W.E. (1986). Review of Clostridium difficile associated diseases. Am. J. Infect. Control. 14: 99 - 109.

18. Paltansing, S.; Guseinova, R.; Berg, R.; Visser, C.; Vorm, E.; Kuijper, EJ. (2007). Characteristics and incidence of Clostridium difficile-associated disease in The Netherlands, 2005. Clin. Microbiol. Infect. 13: 1058 1064.

19. Rudensky, B. Rosoner, S. van Dijk, Y. Shapira, E. (1993). The prevalence and nosocomial acquisition of Clostridium difficile in elderly hospitalized patients. Postgrad. Med. J. 69: 45-47.

20. Rupnik, M.; Grabner, M.; Geric, B. (2003) Binary toxin-producing Clostridium difficile strains. Anaerobe. 9: 289-294.

21. Samore, M.H. (1993). Epidemiology of nosocomial Clostridium difficile infection. Compr. Ther. 19: 151 - 156.

22. Samra, Z.; Talmor, S.; Bahar, J. (2002). High prevalence of toxin A- 
negative toxin B-positive Clostridium difficile in hospitalized patients with gastrointestinal disease. Diagn. Microbiol. Infect. Dis. 43(3): $189-$ 192.

23. Sato, H.; Kato, H.; Koiwai, K.; Sakai, C. (2004). A nosocomial outbreak of diarrhea caused by toxin A-negative, toxin B-positive Clostridium difficile in a cancer center hospital. Kansenshogaku Zasshi. 78(4): 312319.
24. Shin, B.M.; Kim, E.C.; Lee, K.; Kwak, E.Y.; Kang, J.O. (2008). Multicentre study of the prevalence of toxigenic Clostridium difficile in Korea: results of a retrospective study 2000-2005. J. Med. Microbiol. 57: 697-701.

25. Song, J.H.; Shim, K.; Jung, S.; Choi, H.J.; Lee, M.A.; Ryu, K.H.; Kim, S.; Yoo, K. (2008) Antibiotic-associated diarrhea: candidate organisms other than Clostridium difficile. Kor. J. Inter. Med. 23: 9-15. 